\title{
Entstehung, Entwicklung und Zukunft des türkischen Gesundheitswesens
}

Alle nationalen Gesundheitssysteme spiegeln historische Entwicklungen und Erfahrungen wider. Die Türkei blickt auf eine wechselvolle Geschichte zurück, die geprägt ist vom nationalen Befreiungskampf, dem späten Übergang zur westlichen Demokratie und schliesslich den Erfahrungen mit drei Militärinterventionen. All diese Ereignisse hatten auf spezifische Weise Einfluss auf die Entwicklung des Gesundheitsbereichs. Das Land am Bosporus steht gegenwärtig davor, sein Gesundheitssystem für den EU-Betritt zu rüsten. Ankara verspricht sich dadurch die Lösung des medizinischen Versorgungsproblems. Eine Lösung für alle Beteiligten?

\section{Basri Așkın}

\section{Einleitung}

„Beni Türk hekimlerine emanet ediniz“ - zu deutsch: „Gebt mich in die Hände türkischer Ärzte“ lautete einst ein Zitat von Mustafa Kemal Atatürk, dem Staatsgründer der modernen Türkei. Das Zitat drückt auf prägnante Weise die Überzeugung Atatürks und seiner Weggefährten aus, den Sprung in die westliche Moderne neben vielen anderen Bereichen auch in der Gesundheitsfrage zu schaffen. Der Umgang mit sozialen Notlagen der Bevölkerung auf dem Gebiet der heutigen Türkei erfolgte im Osmanischen Reich bis ins 19. Jahrhundert hinein nach den Regeln des Islam (z.B. durch Spenden, Almosen). Die Kranken- und Armenfürsorge wurde hauptsächlich von religiösen, teilweise privaten Stiftungen wahrgenommen, die jahrhundertelang weitgehend unabhängig von staatlicher Einflußnahme wichtige gesellschaftliche, kulturelle und religiöse Aufgaben erfüllten. ${ }^{1}$ Mit der Gründung des türkischen Staates (1923) kam es zu einem radikalen Bruch mit Jahrhunderte alten Traditionen, Werten und Normen im politischen und gesellschaftlichen System. Ausgehend hiervon wird in dem vorliegenden Beitrag auf die Entstehung des modernen türkischen Gesundheitswesens und dessen weitere Entwicklung eingegangen. Die Darstellung rekonstruiert die drei wichtigsten Etappen, die seit 1923 die Entwicklung des medizinischen Systems im Land nachhaltig beeinflusst haben: Frühphase der medizinischen Versorgung (1923-1945), die Sozialisierungsphase nach 1961 sowie schliesslich die neoliberale Umbauphase nach 1980, die seither anhält.

Dipl.-Soz.-Wiss. Basri Așkın, Fachreferent und Autor für soziale Sicherung, Länderschwerpunkt Türkei und Deutschland, Waiblingen
Abgerundet wird der Beitrag mit einer kurzen Zusammenfassung und einem Ausblick auf den sich abzeichnenden Trend in der türkischen Gesundheitspolitik. Der im Zusammenhang mit der aktuellen türkischen Gesundheitsreform vielfach diskutierte Bruch mit dem Status quo erhält so sein bislang fehlendes historisches Glied.

\section{Frühphase der medizinischen Versorgung in der Türkei (1923-1945)}

Mit der Ausrufung der türkischen Republik am 29. Oktober 1923 durch den Staatsbegründer Mustafa Kemal (1881-1938) ging, nach langen und erbitterten Jahren, der nationale Befreiungskampf (1919-1923) gegen die europäischen Besatzungsmächte siegreich zu Ende. Die gesundheitliche Lage bei Republiksgründung war desolat und geprägt durch die hohen Bevölkerungsverluste, die aufgrund des nationalen Befreiungskrieges als auch durch den Ersten Weltkrieg entstanden waren. Viele kehrten verwundet, verstümmelt und invalide aus dem Krieg zurück. Die junge Republik stand vor der Herausforderung, die Folgen des Krieges (wie das starke Ansteigen diverser Infektionskrankheiten, die Folgen der Mangelernährung, die hohe Kindersterblichkeit) sowie die zahlreichen hygienischen Probleme zu bewältigen. Eine führende Rolle spielte dabei Refik Saydam (1881-1942), der als reformorientierter Politiker und enger Weggefährte Atatürks von 1923 an bis zu dessen Tod im Jahre 1937 das Amt des Gesundheitsministers bekleidete. ${ }^{2}$ Als Vorkämpfer für eine öffentliche Gesundheitspflege und eine soziale Medizin war er maßgeblich an der Grundsteinlegung des türkischen Gesundheitswesens beteiligt. Nachfolgend werden die wichtigsten gesundheitlichen Maßnahmen von 1923 bis 1945 kurz dargestellt. 


\subsection{Die Gründung des Ministeriums für Gesundheit und soziale Hilfe (1920)}

Der Ursprung des türkischen Gesundheitswesens reicht zurück bis ins Jahr 1920. Inmitten der Wirren des Befreiungskampfes wurde am 03.05.1920 in einem bescheidenen Arbeitszimmer in Ankara das Ministerium für Gesundheit und soziale Hilfe (»Saḡlık ve Sosyal Yardım Bakanlı $\bar{g}_{1 \ll)}$ eingerichtet. Die Arbeit des Ministeriums bestand Anfangs im Wesentlichen darin, Kriegsverwundete zu versorgen, sowie die Rückführung der so genannten Balkan-Türken ${ }^{3}$ in das Staatsgebiet der heutigen Türkei zu organisieren. Dies ist unter anderem auch ein Grund, weshalb es nicht schlichtweg als Gesundheitsministerium bezeichnet wurde. Nebenbei bemühte man sich noch, mit den wenigen zur Verfügung stehenden finanziellen und personellen Mitteln, die Ausbreitung übertragbarer Krankheiten einzudämmen. Diese Entwicklung weist bereits früh auf die zentrale Bedeutung hin, die der medizinischen Versorgung durch die damalige Landesführung (nicht zuletzt aus militärischen Gründen) beigemessen wurde.

\subsection{Das Allgemeine Gesundheits- und Hygienegesetz (1930)}

Aus der Erkenntnis, dass ohne ausreichende Hygienemaßnahmen die nationale Gesundheitsfrage nicht zu lösen war, wurde im Jahre 1930 das Allgemeine Gesundheits- und Hygienegesetz (»Umumi Hifzissihha Kanunu«) verabschiedet. ${ }^{4}$ Mit diesem Gesetz wurde der landesweite Aufbau der Organisations- und Verwaltungsstruktur des Ministeriums für Gesundheit und soziale Hilfe vorangetrieben und die öffentliche Hygiene zur kommunalen Angelegenheit erklärt. Der Regelungsumfang dieses in weiten Teilen heute noch gültigen Gesetzeswerkes erstreckt sich auf ein weites Feld gesundheitlich relevanter Themenbereiche, angefangen von der Trinkwasserversorgung über die Abfall- und Abwasserbeseitigung und die Totenbestattung, bis hin zum Bau und Betrieb von Gesundheitseinrichtungen (z.B. Krankenhäuser). ${ }^{5} \mathrm{Zu}$ diesem Zweck sah es die Bildung sogenannte Hygiene-Kommissionen auf Provinz- und Bezirksebene vor (»Il ve Ilce Hifzissihha Meclisleri«). Diese waren befugt, in Angelegenheiten, die die öffentliche Gesundheit betrafen, bindende Vorschriften zu erlassen. ${ }^{6}$ Ausserdem wurden mit dem Gesetz grundlegende Fragen der Sicherheit und des Gesundheitsschutzes am Arbeitsplatz geregelt. Beispielsweise wurde die Beschäftigung von Frauen und Kindern eingeschränkt und in größeren Unternehmen die Anstellung von Betriebsärzten (»isyeri hekimleri«) vorgeschrieben. Dies waren frühe Ansätze zur Etablierung einer betrieblichen Gesundheitsversorgung, in deren Folge eine zunehmende Zahl von Versorgungseinheiten auf Unternehmensebene errichtet worden sind. Der Staat beschränkte sich hingegen auf die Seuchenbekämpfung und Prävention und überlies die kurative Versorgung weitgehend den lokalen Verwaltungen, denen er lediglich mit dem Bau entsprechender Musterkrankenhäuser (»Numune
Hastanesi «) landesweit eine wegweisende Richtung vorgab. ${ }^{7}$ Dieses Prinzip bildete den Kern der Saydamschen Gesundheitspolitik, die u.a. auch in dem im Jahre 1930 verabschiedeten neuen Städtegesetz (»Belediye Kanunu«) zum Ausdruck kam. So schrieb das Gesetz unter anderem vor, dass jede Kommune als eigene Rechtsperson verpflichtet sei, für die Gesundheit, Sicherheit und Wohlfahrt ihrer Bürger zu sorgen, die Bettelei zu verbieten, Krankenhäuser zu bauen sowie Apotheken zu eröffnen. ${ }^{8}$

\subsection{Errichtung von Seuchenbekämpfungsdiensten}

Zwecks Verhütung und Bekämpfung von Krankheiten, insbesondere von Infektionskrankheiten bzw. Seuchen (wie z.B. Malaria, Cholera oder Tuberkulose) forcierte Saydam den Aufbau vertikaler Versorgungsstrukturen, die speziell auf die Bekämpfung derartiger Krankheiten ausgerichtet waren. Mit der Zeit bildeten sich so zahlreiche autonome Versorgungsstrukturen in Form von Seuchenbekämpfungsdiensten, die im Zuge der 1936 durchgeführten Organisationsreform des Ministeriums für Gesundheit und soziale Hilfe in dessen Gebilde integriert wurden. ${ }^{9}$ Eine bedeutende Rolle bei der Seuchenbekämpfung spielten die Regierungsärzte (»hükümet tabipleri«), deren Arbeit direkt vom Staat finanziert und von den jeweiligen Provinzgesundheitsbehörden beaufsichtigt wurde. ${ }^{10}$ Die Regierungsärzte wurden ab 1928 mit der Gründung des Refik Saydam Hygiene-Institutes labordiagnostisch unterstützt. Ausserdem setzte Saydam beim Kampf gegen die genannten Hauptgesundheitsprobleme verstärkt auf den Einsatz von Wanderärzten (»Seyyar Tabipler«), die von Dorf zu Dorf gingen und die Bewohner ärztlich versorgten.

\subsection{Die Gründung des nationalen Hygiene-Institutes}

Um Fragen der Hygiene und der Öffentlichen Gesundheit wissenschaftlich $\mathrm{zu}$ thematisieren und $\mathrm{zu}$ erforschen gründete der türkische Staat im Jahre 1928 in Ankara ein nationales Hygiene-Institut (»Hıfzıssıhha Enstitüsü«). Es war damit erstmals imstande, bestimmte Seren und Impfstoffe zu produzieren. Angesichts stetig wachsender Anforderungen wurde der Aufgabenbereich des Hygiene-Institutes in den Folgejahren sukzessiv erweitert. 1942 wurde das Institut zu Ehren seines Begründers in Refik Saydam Hygiene-Institut (RSHI) umbenannt. Das RSHI stellt heute die zentrale Überwachungs- und Forschungseinrichtung der Republik Türkei auf dem Gebiet der „epidemiologischen und medizinischen Analyse und Bewertung von Krankheiten mit hoher Gefährlichkeit, hohem Verbreitungsgrad oder hoher öffentlicher oder gesundheitspolitischer Bedeutung“ dar. Dem Hygiene-Institut ist seit 1937 zusätzlich die Schule für öffentliche Gesundheit (»Hifzissihha Okulu«) als mikrobiologische Lehr- und Weiterbildungseinrichtung angeschlossen. ${ }^{11}$ 


\subsection{Maßnahmen und Herausforderungen im Personalbereich}

Die Lösung der Personal- und Ausbildungsfrage bildete einen weiteren Schwerpunkt von Saydams gesundheitspolitischer Pionierarbeit. Bereits 1928 wurden infolge des Gesetzes Nr. 1219 die wesentlichen Fragen der Arztzulassung und -ausbildung landesweit einheitlich geregelt. Im selben Jahr wurden auch die ersten ärztlichen Vereinigungen gegründet, aus denen später die türkische Ärztekammer hervorging. ${ }^{12}$ Der Staat bemühte sich, den jungen Medizinern im Land, beste Ausbildungsmöglichkeiten zu schaffen. So wurde zur Erhöhung der Absolventenzahlen das Medizinstudium, insbesondere die Ausbildung zum Präventionsarzt, staatlich gefördert. Zusätzlich gelang es der türkischen Regierung nach 1933 unter den deutschen Emigranten für Ankara und Istanbul einen Stab hervorragender Ärzte als Dozenten zu gewinnen. ${ }^{13}$ Ähnlich wurde auch die Ausbildung des nicht-ärztlichen Personals, insbesondere Krankenschwester und Hebammen, staatlich forciert. Trotz intensiver Bemühungen kam es jedoch immer wieder zu Rückschlägen, insbesondere bei der Nachwuchsrekrutierung des weiblichen Gesundheitspersonals. Die strengen gesellschaftlichen Regeln, die in jahrhundertealter islamischer Tradition wurzelten, erlaubte es den Frauen nicht so ohne Weiteres einen Beruf zu erlenen, geschweige den auszuüben. ${ }^{14}$ Aus dieser gesellschaftlichen (Not-)Situation heraus entstand, lange vor Gründung der modernen Türkei, die Berufsgruppe der Gesundheitsbeamten, die nach 1923 eine wichtige Rolle beim Aufbau der medizinischen Versorgung spielte. Die Gesundheitsbeamten haben sich seither als eigenständige Berufsgruppe etabliert und sind heute in vielfältiger Funktion auf allen Ebenen des türkischen Gesundheitswesens vertreten.

\subsection{Die Gründung der Arbeiterversicherungsanstalt}

Ein bedeutender Meilenstein in der Sozialgeschichte des Landes bildet das erste türkische Arbeitsgesetz, welches im Jahre 1936 - noch während der Amtszeit von Refik Saydam - verabschiedet worden ist. Die meisten der zuvor bereits im Allgemeinen Gesundheits- und Hygienegesetz enthaltenen Arbeitsschutzvorschriften wurden aufgehoben und teilweise als neue Rechtsvorschriften in dieses Gesetz integriert. Eine zentrale Forderung des Gesetzes war die Errichtung einer Arbeiterversicherungsanstalt (»Isci Sigortalari Kurumu«). Sie wurde 1945 ins Leben gerufen. Der deutsche Migrant, Prof. Dr. Ernst E. Hirsch (1902-1985), wurde auf der ersten Vollversammlung zu dessen Verwaltungsratsvorsitzenden gewählt. Ziel dieser neuartigen Institution war es, eine für die soziale Sicherheit der Arbeitnehmer erforderliche Struktur zu schaffen. Konkret wurde mit dem Inkrafttreten des Gesetzes Nr. 4772 über die Versicherung bei Arbeitsunfällen, Berufskrankheiten und Mutterschaft am 01.07.1946 der Grundstein hierfür gelegt. ${ }^{15}$ Erwerbstätige Frauen sowie die Ehefrauen versicherter Arbeitnehmer erhielten damit erstmals Leistungen bei Mutterschaft.

\subsection{Zwischenbilanz}

Um die gesundheitlichen Folgen des nationalen Befreiungskampfes zu lindern, wurde staatlicherseits bis zum Ende des Zweiten Weltkrieges fast ausschliesslich in den Aufbau eines primär auf Prävention und Seuchenbekämpfung ausgerichteten Versorgungssystems investiert. ${ }^{16}$ Refik Saydam war als erster Gesundheitsminister des Landes maßgeblich daran beteiligt. Während seiner 14-jährigen Amtszeit stieg die Zahl der Ärzte von 554 auf insgesamt 1538, die der Gesundheitsbeamten von 560 auf 1462. Deutlich stärker entwickelte sich die Zahl der Hebammen, die sich von 136 auf 643 erhöhte. Ein zentraler Posten der Atatürkschen Sozialpolitik bildete dabei das Allgemeine Gesundheits- und Hygienegesetz aus dem Jahre 1930. Sie stellte die Lösung der Gesundheitsfrage erstmals auf eine rationale Grundlage und lieferte einen wichtigen Beitrag zum Aufbau der öffentlichen Hygiene. Daneben wurden, wie schon erwähnt, eine Reihe weiterer wichtiger Gesetze erlassen, wie z.B. das Gesetz Nr. 1219 (Ärztliche Approbationsordnung) oder das Organisationsgesetz Nr. 3017 des Ministeriums für Gesundheit und soziale Hilfe aus dem Jahre 1936, die allesamt die Basis für den Aufbau einer landesweiten Versorgungsinfrastruktur lieferten. Die Rolle der Städte und Gemeinden war hauptsächlich auf die Sicherstellung der kurativen medizinischen Versorgung der lokalen Bevölkerung ausgerichtet. Obwohl sich der Gesundheitszustand der Bevölkerung allgemein verbesserte, traten immer wieder Seuchen- und Infektionskrankheiten auf, die zu großen Verlusten in der Bevölkerung führten. Allein im Jahre 1945 forderte eine Malaria-Epidemie hunderttausende Opfer im Land, woraufhin im selben Jahr ein spezielles Gesetz zur Bekämpfung der Malaria verabschiedet wurde.

\section{Die Zeit von 1946-1960}

Die Periode zwischen 1946 bis 1960 ist sowohl innenwie aussenpolitisch von großer Bedeutung. Innenpolitisch wurde 1946 mit dem Übergang vom Einparteienzum Mehrparteiensystem formal die Demokratie eingeführt. Atatürks Republikanische Volkspartei (CHP) wurde schliesslich bei den Wahlen 1950 von der konservativen Demokratischen Partei (DP), die mit ihrer liberalen Wirtschaftsauffassung die gesamten fünfziger Jahre politisch dominierte, auf die Oppositionsbank gedrängt. Aussenpolitisch entschied sich die Türkei im Zuge des sich abzeichnenden Ost-West-Konflikts für eine Allianz mit dem Westen, womit es endgültig seinen von Staatsgründer Atatürk verordneten Kurs der Neutralität aufgab. ${ }^{17}$ Im gesundheitlichen Bereich befand sich die Türkei unter den Gründungsmitgliedern der im Jahre 1948 ins Leben gerufenen Weltgesundheitsorganisation (WHO). Zur selben Zeit war das Ministerium für Gesundheit und soziale Hilfe bemüht, einen 10 Jahre umfassenden nationalen Gesundheitsplan zu verabschieden, welcher die Einführung eines beitragsfinanzierten Krankenversicherungssystems vorsah. Der Plan wurde jedoch nie umgesetzt. ${ }^{18}$ Die Vorstellung, die Gesundheitsfrage via Krankenversicherung für alle einheitlich zu lösen, war angesichts des Entwick- 
lungsstadiums der damaligen Türkei schlichtweg unrealistisch. ${ }^{19}$ Stattdessen wurde ein gänzlich anderer Entwicklungspfad eingeschlagen, der primär die schrittweise medizinische Absicherung einzelner Berufsgruppen im Visier hatte.

\subsection{Die medizinische Absicherung der Beamten}

Die Beamtenschaft bildete die Bevölkerungsgruppe in der Türkei, deren medizinische Absicherung als erste und am umfassendensten geregelt wurde. Mit der Gründung der Pensionskasse (»Emekli Sandiḡ $i \ll)$ im Jahre 1949 wurden die bereits bestehenden Unterstützungskassen für diverse Beamtengruppen (z.B. Soldaten, Offiziere, Lehrer) aufgelöst und eine einheitliche soziale Absicherung der Staatsbediensteten und ihrer Familienangehörigen gewährleistet. ${ }^{20}$ Die medizinische Versorgung der Beamtenschaft erfolgte anfangs überwiegend in den zum Teil eigens dafür errichteten Städtischen Krankenhäusern (»Belediye Hastaneleri «). ${ }^{21}$ Die Pensionskasse übernahm die Kosten für die medizinische Versorgung der Beamten im Alter. Aktive Beamte wurden und werden dagegen direkt über ihre öffentlichen Arbeitgeber versichert. Die traditionell privilegierte Stellung des Militärs und der Beamten, die als Staatselite die Verwestlichung von Staat und Gesellschaft traditionell am stärksten vorantreiben sollte, kam somit auch in der medizinischen Absicherung zum Ausdruck.

\subsection{Arbeiterkrankenversicherung}

Auf der Grundlage des Gesetzes Nr. 5502 wurde im Jahre 1951 die Arbeiterkrankenversicherung als neuer Versicherungszweig im Rahmen der Arbeiterversicherungsanstalt eingeführt. ${ }^{22}$ Die landesweite Umsetzung der entsprechenden Rechtsvorschriften erfolgte - ausgehend vom europäischen Teil der Türkei (Istanbul, östliches Thrakien) - schrittweise bis etwa 1960 auf nahezu sämtliche Landesteile mit industrieller Ansiedlung. Parallel dazu wurde aus Beitragsmitteln der Arbeitnehmer und Arbeitgeber eine arbeitnehmereigene Versorgungsinfrastruktur finanziert und aufgebaut, was zu teilweise erheblichen Spannungen zwischen dem Arbeitsministerium und dem damaligen Ministerium für Gesundheit und soziale Hilfe führte, da letztere die alleinige Versorgungshoheit für sich beanspruchte. Wegen seiner erst im Aufbau befindlichen Gesundheitsinfrastruktur war das Gesundheitsministerium jedoch nicht imstande eine ausreichende medizinische Versorgung der Arbeiterschaft zu gewährleisten. Davon abgesehen war das Arbeitnehmerlager von Beginn an für die Errichtung eines eigenständigen Versorgungssystems. Diese frühe strategische Richtungsentscheidung der Arbeiterversicherungsanstalt hat die sozial- und gesundheitspolitische Debatte in der Türkei bis heute nachhaltig geprägt.

\subsection{Mutter-Kind-Fürsorge}

Abgesehen vom Bau vereinzelter Geburts- und Kinderbetreuungshäuser («Doğum ve Çocuk Bakımevleri») wurde der Mutter-Kind-Fürsorge im Land bis 1950 keine besondere Aufmerksamkeit geschenkt. Im Jahre 1952 begann der türkische Staat mit Hilfe der WHO und der UNICEF eine autonome Struktur zur Mutter-Kind-Fürsorge mit landesweiten Mutter-Kind-Zentren aufzubauen. Der Verantwortungsbereich des Ministeriums für Gesundheit und soziale Hilfe wurde damit um eine weitere wichtige Säule erweitert. Diese Entwicklung führte dazu, dass der Bedarf an Kinderärzten zunahm, aber nicht gedeckt werden konnte. Zeitweise wurden in den MutterKind-Zentren aus Mangel an Kinderärzten Allgemeinmediziner beschäftigt, die durch Krankenschwestern und Hebammen entsprechend unterstützt wurden.

\subsection{Die Verstaatlichung der Krankenhausversorgung}

Nachdem der türkische Staat die Krankenhausversorgung jahrzehntelang als lokale Angelegenheit betrachtet hatte, begann er im Jahre 1954 einen Großteil der Städtischen Krankenhäuser sowie zahlreiche Stiftungskrankenhäuser (mit Ausnahme der SSK-Krankenhäuser) zu verstaatlichen. Der Sicherstellungsauftrag für die stationäre Versorgung ging damit erstmals auf den Staat über. Parallel dazu wurde in den Bau zusätzlicher Krankenhäuser (»devlet hastaneleri«) investiert. Das staatliche Interesse an der stationären Versorgung ließ die Krankenhäuser, insbesondere auch wegen der besseren Verdienstmöglichkeiten, zu neuen und vor allem bevorzugten Beschäftigungsorten für das Gesundheitspersonal werden. Die Folge war eine zunehmende Konzentration des Gesundheitspersonals in den Städten, und eine sich verstärkende Tendenz zur Spezialisierung innerhalb der Ärzteschaft. Beide Aspekte stärkten deren Selbstbewusstsein. Dieses Selbstbewusstsein wurde bereits im Vorfeld mit der Gründung der türkischen Ärztekammer (»Türk Tabipleri Birliḡi $\ll$ ) im Jahre 1953 öffentlich demonstriert. ${ }^{23}$ Der nach wie vor bestehende Mangel an Pflegepersonal veranlasste den Gesetzgeber im Jahr darauf das türkische Krankenpflegegesetz zu verabschieden.

\subsection{Zusammenfassung}

Die Periode zwischen 1946 und 1960 war für die weitere Entwicklung des türkischen Gesundheitswesens von zentraler Bedeutung, wurden doch mit der Arbeiterkrankenversicherung und dem Einzug des Staates in die Krankenhausversorgung in den fünfziger Jahren entscheidende Weichenstellungen vorgenommen. Infolge der Einführung der Arbeiterkrankenversicherung geriet der Gedanke an die betriebliche Gesundheitspolitik, wie sie das Allgemeine Gesundheits- und Hygienegesetz vorgesehen hatte, in Vergessenheit. Arbeitnehmer und Arbeitgeber waren mehrheitlich der Auffassung, dass sie keine geeignete Stragie zur Lösung der nationalen Gesundheits- 
frage sei. Das Konstrukt der türkischen Arbeiterkrankenversicherung war äußerst umstritten, weil es die Mehrheit der türkischen Bevölkerung de facto vom Zugang zur medizinischen Versorgung ausschloss. Die staatliche Haltung in der Gesundheitsfrage blieb, trotz des punktuellen Eindringens in die kurative Versorgung (z.B. Krankenhausbereich), nach wie vor auf die Verhütung und Bekämpfung ansteckender Krankheiten beschränkt. ${ }^{24}$ Dies änderte sich nach dem Militärputsch am 27. Mai 1960 grundlegend. Die Militärintervention von 1960 war die Folge auf den repressiven Machtmissbrauch der DP-Regierung, die mit ihrer Liberalisierungspolitik das Land im Verlauf der fünfziger Jahre immer stärker in die Schuldenfalle gestürzt hatte.

\section{Die Zeit von 1961-1980}

Nach dem gescheiterten Mehrparteienversuch glaubte das türkische Militär die Lösung in einer stärkeren Demokratisierung und Selbstkontrolle der Macht gefunden zu haben. ${ }^{25}$ Die vom Militär ausgearbeitete neue Verfassung von 1961 weitete die Grundrechte aus und führte zu einer freiheitlich-liberalen Aufbruchstimmung im Land. ${ }^{26}$ Im sozialen Bereich prägte die Aufnahme des Sozialstaatsgedankens in die Verfassung die weitere Entwicklung. Den Höhepunkt bildete dabei zweifelsohne das in Artikel 49 verankerte Recht auf Gesundheit. Die bestehenden Versorgungsstrukturen boten keine Grundlage die medizinische Versorgung der breiten Bevölkerungsmassen, die nach wie vor auf dem Land lebte, ausreichend sicherzustellen. Die wenigen Krankenhäuser befanden sich hauptsächlich in den Großstädten. Die Versorgung ausserhalb der Städte hingegen war kaum entwickelt. Urbanisierung und Landflucht trugen ihrerseits als Folgeerscheinungen des sich abzeichnenden Modernisierungsprozesses zu einer Verschärfung der Situation bei. Was die Regierungsärzte anging, waren sie den gesundheitlichen Herausforderungen nicht mehr gewachsen. Ihre Tätigkeit beschränkte sich im Zeitablauf immer mehr auf die Administration und Rechtsmedizin. Dies, sowie die erneute Rückbesinnung des Landes auf eine geplante Wachstums- und Entwicklungsstrategie, führte zu einem fundamentalen Richtungswechsel und damit gleichzeitig zum endgültigen Bruch mit der Saydamschen Gesundheitspolitik. ${ }^{27}$

\subsection{Das Sozialisierungsgesetz Nr. 224}

Aus der Erkenntnis, dass ohne ausreichende medizinische Infrastruktur weder eine nachhaltige Verbesserung des allgemeinen Gesundheitszustandes, noch eine positive wirtschaftliche Entwicklung möglich war, wurde im Jahre 1961 der Aufbau des öffentlichen Gesundheitswesens eingeleitet. ${ }^{28}$ Grundlage bildete das Gesetz Nr. 224 vom 05.01.1961. Seine Intention war die landesweit flächendeckende Bereitstellung von Gesundheitsdiensten, die unter der Bezeichnung »Sozialisierung der Gesundheitsdienste« die Verstaatlichung des Gesundheitswesens zur Folge hatte. ${ }^{29}$ Basierend auf einem Gesundheitsbegriff, welcher in Anlehnung an die WHO-Definition Gesundheit nicht nur als die bloße Abwesenheit von Krankheit, Gebrechen und Invalidität ansah, sondern auch das geistige und soziale Wohlbefinden berücksichtigte, sollte es den freien und gleichberechtigten Zugang aller zur medizinischen Primärversorgung in die Tat umsetzen. ${ }^{30}$ Charakteristisch für die sozialisierten Gesundheitsdienste sind ihre Diskriminierungsfreiheit, ihre Zugänglichkeit und ihre Erschwinglichkeit. Ausdruck der Sozialisierung war auch, dass die privatärztliche Tätigkeit der in die sozialisierten Gesundheitsdienste involvierten Ärzte vom Gesetzgeber explizit ausgeschlossen wurde. Nusret Fişek (1914-1990) hat als führender Arzt und Politiker des Landes die Grundsätze der Sozialisierung nachhaltig geprägt. Diese sind z.B.:

- Kostenloser Zugang zur medizinischen Primärversorgung

- Integration von kurativen und präventiven Maßnahmen unter einem Dach

- „Vorbeugen statt Heilen“

- Gestufte Versorgung (Überweisungssystem)

- Partizipation der Bevölkerung in das lokale Versorgungsgeschehen

- Berufsübergreifender Versorgungsansatz (Teamwork)

- Harmonisierung der Gesundheitsdienste (keine Doppelund Mehrfachstrukturen)

Das Gesetz Nr. 224 stellt ein bis heute gültiges Gesetz dar, das über die Schaffung einer nachhaltigen und effizienten Primärversorgung hinaus den gesundheitspolitischen Ordnungsrahmen vorgab und dabei zugleich die allgemeinen Prinzipien der Gesundheitsversorgung festlegte. Trotz gewisser Ähnlichkeit mit einem nationalen Gesundheitsdienst (wie z.B. in Großbritannien, Italien) unterscheidet sich die Systemlogik im Fall des türkischen staatlichen Gesundheitsdienstes, der die ambulante und stationäre Versorgung umfasst, in zwei Punkten wesentlich vom britischen National Health Service (NHS). Erstens in der Art der Leistungsfinanzierung und zweitens in der generellen Konzeption des türkischen Pendants als reiner Leistungsanbieter ohne integrierte Versicherungskomponente: Das türkische Gesundheitsministerium ist kein Krankenversicherungsunternehmen (wie das NHS), sondern hält die notwendige Gesundheitsinfrastruktur die prinzipiell jedermann zugänglich ist - landesweit vor. Hinsichtlich der Art der Finanzierung weist das Gesetz Nr. 224 ausdrücklich auf einen Mix aus Steuern, Beiträgen und Eigenbeteiligungen hin. Ein nationaler Gesundheitsfonds ist nicht vorgesehen. Daraus resultiert unmittelbar das sogenannte Sozialisierungsparadox: der Staat verfolgt einerseits das Ziel „medizinische Versorgung für alle" und investiert in die Errichtung einer öffentlichen Gesundheitsinfrastruktur, deren Betreiber er zugleich ist. ${ }^{31}$ Andererseits wird der Zugang zu den öffentlichen Gesundheitseinrichtungen über ein berufsgruppenspezifisches Krankenversicherungssystem gesteuert, von der allerdings Viele nicht erfasst sind. 


\subsection{Das Gesundheitsposten-Modell}

Die Grundsätze der Sozialisierung bilden sich im sogenannten Gesundheitsposten-Modell ab. Bei diesem Modell repräsentieren die Gesundheitsposten (»Saḡlık Ocaklan«) die zentralen Primärversorgungseinrichtungen. Sie sind als integrierte und interdisziplinäre Einheiten, für eine bestimmte Anzahl von Menschen, als erste Anlaufstelle bei Beschwerden gedacht und mit zahlreichen Einrichtungen des staatlichen Gesundheitsdienstes vernetzt. Es handelt sich dabei um ein zweistufiges Versorgungsmodell (siehe Abbildung 1). ${ }^{32}$ Auf der Primärversorgungsebene stellt die Integration von Gesundheitsförderung, -beratung, Präventionsprogrammen (z.B. Impfungen) und kurativer Medizin (Erste Hilfe, Bekämpfung endemischer Krankheiten) ein zentrales Element der Versorgung dar. Das Modell wurde erstmals im Jahre 1963 in der südöstlichen Provinz Muş umgesetzt und in der Folgezeit sukzessiv auf alle übrigen Provinzen des Landes übertragen. Im selben Jahr wurde die Regierungsärzteschaft aufgelöst. städten. Die Distriktkrankenhäuser sind für die ländlichen Bevölkerung beinahe die einzigste Möglichkeit, Krankenhausleistungen in Anspruch zunehmen. Es handelt sich um ein hierarchisches Modell, bei dem das Ministerium in Ankara die Zielrichtung vorgibt. Die Provinzgesundheitsbehörden sind in den einzelnen Provinzen zwar für die Gesundheitsversorgung zuständig, besitzen aber keine nennenswerte Planungs- und Budgetkompetenzen.

\subsection{Familienplanung als staatliche Aufgabe}

In der Verfassung von 1961 wurden Schutz und Förderung der Familie erstmals in der Türkei als Staatsaufgabe definiert. ${ }^{34}$ Angesichts der hohen Verbreitung ungewollter Schwangerschaften, Selbstabtreibungen und der damit verbundenen Gefahr für Mutter und Kind, wurden auf der Grundlage des Gesetzes Nr. 557 vom 01.04.1965 die Leistungen zur Familienplanung geregelt. Ziel war es, die medizinische Betreuung für Mutter und Kind langfristig mit der Beratung zu Fragen und Möglichkeiten der Familienplanung zu verknüpfen. Konkret umfassten die Maßnahmen die kostenlose ärztliche Beratung über Fragen der Empfängnisverhütung, einschliesslich der erforderlichen Untersuchung und der Verordnung von empfängnisregelnden Mitteln. Angesichts des rapiden Bevölkerungswachstums, der sich vor allem nach 1960 in den Städten zeigte, fand ein Umdenken in der bisherigen Bevölkerungspolitik statt. ${ }^{35}$ Sowohl im Hinblick auf die wirtschaftliche Entwicklung des Landes, als auch unabhängig von der Intention, das Bevölkerungswachstum zu verlangsamen, wurde die Bedeutung von Familienplanung als Teil einer ganzheitlich angelegten Präventionsstrategie erkannt. Mit dem Einwirken des Staates auf das reproduktive Verhalten, sollte nicht zuletzt die Modernisierung der Familie vorangetrieben werden. ${ }^{36}$
Wie in Abbildung 1 ersichtlich, nehmen die Gesundheitsposten innerhalb der staatlichen Primärversorgung eine Schlüsselposition mit zugewiesener Lotsenfunktion ein. Die Gesundheitshäuser (»Saḡlık Evleri«) bilden dabei eine Untereinheit der Gesundheitsposten und sind entsprechend verantwortlich für die Basisversorgung in den entlegenen Dörfern. Daneben gibt es die Dispensaire (»Dispanser «), von denen die sog. Tuberkulose-Dispensaire (»Verem Savas Dispanserleri«) am bekanntesten sind. Ihre Aufgabe ist die zielgerichtete Präventions- und Therapiearbeit mit Tuberkulose-Patienten (z.B. Aufklärung, medikamentöse Behandlung). ${ }^{33}$ Unterstützt wird die Primärversorgung schliesslich durch die Volksgesundheitslaboratorien (»Halk saḡlıḡı laboratuvarları«). Ergänzt wird die Primärversorgung auf der nächsthöheren Ebene durch die Distrikskrankenhäuser und schliesslich durch die großen Zentralkrankenhäuser in den Provinzhaupt-

\subsection{Die medizinische Versorgung Älterer}

Die Symbiose zwischen staatlichem Gesundheitsdienst und beitragsfinanzierter Sozialversicherung war nicht im Stande, alle Bevölkerungsgruppen (z.B. Arbeitslose, Saisonarbeiter, Tagelöhner) in das soziale Netz einzubinden. Dazu zählten insbesondere auch ältere Menschen. Sie waren oft ohne jegliche soziale Absicherung, weder im Rahmen der Sozialversicherung noch im familiären Umfeld. Der türkische Staat griff diese Problematik auf und verabschiedete am 10.7.1976 ein Gesetz, das bedürftigen Menschen ab 65 Jahren ein Anrecht auf finanzielle staatliche Existenzsicherung gewährte. Damit verbunden war der Anspruch auf kostenlose medizinische Behandlung in den staatlichen Gesundheitseinrichtungen. Seitdem 
gibt es zahlreiche solcher Hilfsprogramme, die überwiegend aus Steuermitteln finanziert werden. Der Staat tritt in der Regel nur über sie direkt als Leistungsfinanzierer in Erscheinung (siehe Kapitel 6.1). ${ }^{37}$

\subsection{Das Sozialversicherungsgesetz Nr. 506}

Rund 20 Jahre nach Gründung der Arbeiterversicherungsanstalt erlebte die Arbeitnehmerversicherung mit der Verabschiedung des Sozialversicherungsgesetzes Nr. 506 am 17.07.1964 seine erste umfassende Reform. Sämtliche Einzelgesetze, die nach und nach für die soziale Sicherung der Arbeitnehmer verabschiedet worden waren, wurden aufgehoben und gingen als neue Rechtsvorschriften in dieses Gesetz ein. ${ }^{38}$ Auf der Ausgabenseite wurde die ambulante (zahn-)ärztliche Versorgung (einschliesslich Arzneimittel), stationäre Behandlung und medizinische Rehabilitation um Krankengeldleistungen sowie Maßnahmen im Bereich der Gesundheitsförderung und Prävention ergänzt. ${ }^{39}$ Problematisch war allerdings in der Folgezeit die sukzessive Ausweitung des Versichertenkreises auch auf die verkammerten Berufe (z.B. Rechtsanwälte, Architekten, Notare), während insbesondere die soziale Absicherung der landwirtschaftlichen Arbeitnehmer lange Zeit ungeregelt blieb (siehe Punkt 5.1). Ein weiteres Ergebnis dieser Reform war 1964 die Umbenennung der Arbeiterversicherungsanstalt in Sozialversicherungsanstalt (SSK), die mit der deutschen AOK vergleichbar ist. Im Zuge der türkischen Arbeitsmigration nach Deutschland wurde die SSK 1964 im Rahmen des deutsch-türkischen Sozialversicherungsabkommens zum offiziellen Vertragspartner auf türkischer Seite erklärt.

\subsection{Die gesetzliche Krankenversicherung der Selbständigen (Baḡ-Kur)}

Mit der Errichtung der Pensionsversicherungsanstalt (Baḡ-Kur) im Jahre 1971 erhielt das türkische Sozialversicherungssystem seinen letzten großen Versicherungsträger. Sie ist seither zuständig für die soziale Absicherung der Selbstständigen und Freiberufler. Die zunehmenden wirtschaftlichen und sozialen Wandlungsprozesse brachten es mit sich, dass über die traditionellen Gruppen der Beamten und Arbeitnehmer hinaus, eine Ausweitung der sozialen Sicherung auch auf Selbständige und Freiberufler für erforderlich gehalten wurde. Sowohl die Pensionskasse als auch die Pensionsversicherungsanstalt wurden im Gegensatz zur SSK als reine Kostenträger konzipiert.

\subsection{Zwischenbilanz}

Bis 1950 hat die türkische Gesundheitspolitik hauptsächlich der Bekämpfung von Infektionskrankheiten Vorrang eingeräumt. Während der fünfziger Jahre wird diese einseitige Ausrichtung in der Gesundheitspolitik brüchig. Als im Jahre 1961 mit dem Begriff Sozialisierung der Gesundheitsdienste der Startschuss für den Aufbau des öffentlichen Gesundheitswesens fällt, führt dies entgegen der Absicht der Sozialisierungsbefürworter zu einer Zweiteilung des Gesundheitswesens mit dem staatlichen Gesundheitsdienst auf der einen und dem arbeitnehmereigenen SSK-Versorgungssystem auf der anderen Seite (duale Versorgung). ${ }^{40}$ Diese Dualität war bereits gegen Ende der siebziger Jahre voll ausgebildet. ${ }^{41}$ Mangelnder politischer Wille und chronische Unterfinanzierung führten dazu, dass das großangelegte Reformprojekt zur Sozialisierung der Gesundheitsdienste bereits nach kurzer Zeit ins Stocken geriet. Zu groß erschien die Kluft zwischen dem Sozialstaatsgedanken der Verfassung von 1961 und der Realität in der türkischen Gesellschaft. Nach 1970 wurde zudem die türkische Wirtschaft immer mehr dazu gedrängt, sich auf privatkapitalistischer Basis in den Weltmarkt zu integrieren. Wegen erneuter politischer und sozialer Unruhen putschte das Militär 1971 zum zweiten Mal, wodurch das Land ein weiteres Mal in seiner Geschichte politisch lahm gelegt wurde. Die steigende Auslandsverschuldung führte zu einer stärkeren Abhängigkeit des türkischen Staates durch die ausländischen Kreditgeber. Bis auf die Gründung der Baḡ-Kur konnten in dieser Dekade keine nennenswerten Gesundheitsreformmaßnahmen das türkische Parlament passieren.

\section{Die 1980er Jahre}

Einen historischen Wendepunkt in der türkischen Wirtschaft bildeten die Beschlüsse vom 24. Januar 1980, die das Ende des Protektionismus in der Wirtschaftspolitik des Landes einleitete. Sie war einer der Hauptgründe für den Militärputsch vom 12. September 1980. Während der Militärherrschaft (1980-1983) wurden die Forderung des Internationalen Währungsfonds nach einer rückhaltlosen Öffnung der türkischen Wirtschaft durchgesetzt. Der neoliberale Kurswechsel hatte auch unmittelbare Auswirkungen auf die Gesundheitspolitik des Landes. Die Verfassung von 1961 wurde durch die Verfassung von 1982 ersetzt und die Allgemeine Krankenversicherung erstmals als mögliche Finanzierungs- und Bereitstellungsoption medizinischer Leistungen in der Verfassung verankert. Das Recht auf Gesundheit wurde gestrichen und Gesundheit zu einer Leistung degradiert, die der Staat fortan lediglich im Rahmen seiner finanziellen Möglichkeiten bereitzustellen verpflichtet war. Vor diesem Hintergrund nahm das Ministerium für Gesundheit und soziale Hilfe, das seit 1988 Gesundheitsministerium heißt, gegen Mitte der achtziger Jahre die ersten Gespräche mit der Weltbank auf.

\subsection{Die Krankenversicherung der Landwirte und der Saisonarbeiter}

Ein wichtiges gesundheitspolitisches Ereignis der achtziger Jahre bildete 1983 die Aufnahme der Landwirte in das Sozialversicherungssystem. Ein Jahr später wurde unter dem Dach der Pensionsversicherungsanstalt die Kran- 
kenversicherung der Landwirte eingeführt. Sie war eine verspätete Antwort des Staates auf die Sorgen und Nöte der ländlichen Bevölkerung. Schliesslich stellte und stellt die Landwirtschaft, wenn auch mit abnehmender Tendenz, einen bedeutsamen Wirtschafts- und Beschäftigungsbereich dar. Im selben Jahr wurde auf freiwilliger Basis die soziale bzw. medizinische Absicherung der einheimischen Saisonarbeiter in der Landwirtschaft geregelt, nachdem bereits zuvor die unbefristet Beschäftigten dieses Sektors im Rahmen der SSK in die Pflichtversicherung aufgenommen worden waren. Die berufsgruppenspezifischen Ausweitung der Krankenversicherung war letztlich die Folge jener Überzeugung, die primär die gesundheitliche Absicherung der erwerbstätigen Bevölkerung im Auge hatte.

\subsection{Das Gesundheitsgesetz aus dem Jahre 1987}

Die bestehenden Rahmenbedingungen im Gesundheitswesen boten vor 1980 keinerlei Anreize für privatwirtschaftliche Aktivitäten. Als größtes Hindernis erwies sich dabei die Regelung, die den Ärzten im öffentlichen Gesundheitswesen eine privatärztliche Tätigkeit untersagte. Dies änderte sich infolge der Verfassung von 1982 und der anschliessenden Verabschiedung des Gesundheitsgesetzes (»Temel Saḡlık Hizmetleri Kanunu«) im Jahre 1987 mit der die ersten konkreten Schritt in Richtung einer marktwirtschaftlichen Öffnung des zentralistisch organisierten türkischen Gesundheitswesens erfolgten. ${ }^{42}$ Und das bedeutete in erster Linie die Einführung betriebswirtschaftlicher Elemente in die staatlichen Versorgungseinrichtungen. Neben der Möglichkeit zur Schließung bzw. Zusammenlegung nicht ausgelasteter Einrichtungen wurde infolge dieses Gesetzes den verbeamteten Ärzten erstmals erlaubt, nach Dienstschluss erbrachte ärztliche Leistungen privat abzurechnen. Dies bedeutete für die staatlichen Krankenhäusern eine neue Einnahmequelle, die der türkische Gesetzgeber den Krankenhäusern in begrenztem Umfang zur eigenverantwortlichen Verwendung überlies. Eine weitergehende Regelung, die die Beschäftigung von in- und ausländischen Ärzten mit spezifischen Fachkenntnissen in den staatlichen Gesundheitseinrichtungen auf arbeitsvertraglicher Basis ermöglichen sollte, wurde vom türkischen Verfassungsgericht für nichtig erklärt.

\subsection{Die Master-Plan-Studie zum Gesundheitssektor}

Um die bestehenden Defizite im türkischen Gesundheitswesen offenzulegen und hierauf basierend erste Lösungsvorschläge zu erarbeiten, wurde unter der Federführung der staatlichen Planungsbehörde (DPT) in Zusammenarbeit mit ausländischen Experten im Jahre 1988 mit der Erstellung eines „Masterplans Gesundheit“ begonnen. Der Plan, der 1990 abgeschlossen und seither von nahezu allen türkischen Regierungen in der Vergangenheit als Rahmenwerk für „eigene“ Reformvorschläge genutzt wurde, bemängelte insbesondere
- das Nebeneinander zweier abgeschotteter Versorgungssysteme (duale Versorgung),

- die regionalen Unterschiede in der bestehenden Gesundheitsversorgung (West-Ost-Gefälle),

- den Personalmangel (sowohl in quantitativer als auch qualitativer Hinsicht) sowie

- den niedrigen Ausgabenanteil für Gesundheit

Man war sich einig, dass die Probleme hauptsächlich von den bestehenden Strukturen herrührten. Statt einer grundlegenden Strukturreform wurde im Master-Plan die Ergänzung des bestehenden Gesundheitssystems um marktwirtschaftliche Elemente favorisiert.

\subsection{Folgen und Auswirkungen}

Die achtziger Jahre waren in nahezu jeder Hinsicht durch die Folgen der Militärherrschaft geprägt, die die weitgehende Entpolitisierung der Gesellschaft zum Ziel hatte. Aus den Erfahrungen mit der türkischen Studentenbewegung, die in den sechziger und siebziger Jahren ihren Höhepunkt erreicht hatte, wurde 1981 der türkische Hochschulrat (»YÖK«) als verfassungsmäßige Institution etabliert. Sämtliche Hochschulen und Universitäten des Landes (einschliesslich der Universitätsklinken) sind seither dem YÖK unterstellt, dessen Aufgaben und Zuständigkeiten sich sowohl auf personelle wie auch inhaltliche und organisatorische Entscheidungen der Universitäten erstreckt. Parallel zur Ausweitung der staatlichen Kontrolle wurde die Privatwirtschaft aufgebaut. So kam es in der Folgezeit zu zahlreichen Versorgungsarrangements sowohl des Gesundheitsministeriums als auch der SSK mit dem privaten Sektor. Während der „private“ Sektor vor 1980 allenfalls rudimentär existierte und sich überwiegend in den Händen einiger weniger Ausländer und religiöser Minderheiten befand, entwickelte er sich nach $1980 \mathrm{zu}$ einem lukrativen Markt für privatwirtschaftliche Investitionen.

\section{Die 1990er Jahre}

Die im Zuge der Sozialisierung eingeleitete Verstaatlichung des Gesundheitswesens geriet in den neunziger Jahren zunehmend unter Legitimationsdruck. Als Begründung wurden insbesondere die globalen Änderungen in der Wirtschaft sowie die stetig steigenden Ausgaben im türkischen Gesundheitswesen herangezogen. Der staatliche Anteil im Bereich Gesundheit machte Anfang der neunziger Jahre mit 61,9 \% bereits über die Hälfte der gesamten Ausgaben im Gesundheitssektor aus.

\subsection{Der erste nationale Gesundheitskongress (1992)}

Um das türkische Gesundheitswesen zukunftsfähig zu machen und die bestehenden Defizite abzubauen, versammelten sich unter der Federführung des Gesundheitsministeriums in Ankara vom 23.03.1992 bis 27.03.1992 
hochrangige Vertreter zum Ersten Nationalen Gesundheitskongress. Auf diesem Kongress wurde der Status quo im türkischen Gesundheitswesen ausführlich thematisiert und ein konkreter Reformvorschlag aus Sicht des Gesundheitsministeriums als Arbeitsgrundlage vorgestellt. Wichtige Forderungen waren dabei u.a.

- der Ausbau der medizinischen Infrastruktur, insbesondere im Osten des Landes,

- die Dezentralisierung des nationalen Gesundheitsdienstes,

- die Schaffung besserer Anreize für das Gesundheitspersonal,

- die verstärkte Zusammenarbeit mit internationalen Organisationen auf dem Gebiet der Gesundheit,

- der Aufbau eines Gesundheitsinformationssystems zur Gewinnung regionaler Gesundheitsinformationen,

- die bessere Vernetzung und Kooperation aller an der Gesundheitsversorgung Beteiligten.

Bereits Ende 1991 wurde mit finanzieller Unterstützung der Weltbank das erste Gesundheitsprojekt gestartet, das zur Verbesserung der Gesundheitsversorgung in den unterentwickelten Regionen, insbesondere im Osten des Landes, beitragen sollte. Drei Monate nach dem Ersten Gesundheitskongress wurde deshalb am 18.6.1992 die Grüne-Karte-Regelung erlassen, die bedürftigen Menschen den Zugang zu (kostenloser) medizinischer Behandlung in den staatlichen Gesundheitseinrichtungen eröffnete. Gegenwärtig sind knapp 11 Millionen Bürger im Besitz einer Grünen Karte. Daneben gibt es für „Bedürftige" die Möglichkeit den Sozialhilfe- und Solidaritätsfond zu beanspruchen, welche nicht durch die Grüne Karte abgedeckte Kosten übernimmt (z.B. Medikamente, Prothesen).

\subsection{Zweiter Nationaler Gesundheitskongress (1993)}

Der mit dem Masterplan Gesundheit und dem anschliessenden Treffen der Beteiligten auf dem ersten nationalen Gesundheitskongress ins Rollen gebrachte Gesundheitsreformdiskurs hatte bereits auf dem zweiten nationalen Gesundheitskongress, der vom 12. bis 16. April 1993 in Ankara abgehalten wurde, zu einem ersten Kompromiss zwischen den Beteiligten aus Politik, Gesundheit, Wirtschaft und Wissenschaft geführt. Mehrheitlich gingen Themen wie Allgemeine Krankenversicherung und Hausarztmodell als mögliche Lösungsvorschläge in die engere Auswahl ein. Es stellte sich heraus, dass insbesondere Fragen nach Möglichkeiten zur Verbesserung des Gesundheitszustand der Bevölkerung unter Berücksichtigung von Wirtschaftlichkeitsaspekten bzw. die Forderung nach einem gleichberechtigten Zugang zur medizinischen Versorgung für alle und ähnlicher Fragen verstärkt anzugehen sind. In diesem Zusammenhang wurden 1995 mehrere Gesetzentwürfe seitens der Regierung ins Parlament eingebracht, deren Vorschriften nach der Verabschiedung schrittweise im Land umgesetzt werden sollten. Dazu zählten u.a. das Gesetz zur Einführung des Hausarztmodells sowie das Krankenhausneuord- nungsgesetz. Aufgrund der vorzeitigen Wahlen im Jahr 1995 konnten die Gesetzentwürfe jedoch nicht verabschiedet werden. Das Thema Gesundheitsreform wurde erst nach der Wahl wieder aufgegriffen, diesmal allerdings im Rahmen des 7. Fünfjahresplans, der den Zeitraum von 1996 bis 2000 umfasste. Trotz rasch wechselnder Regierungen in der Folgezeit blieben die Themen im Gesundheitsbereich dieselben. 1997 und 1998 wurden dem Parlament erneut entsprechende Gesetzentwürfe zur Allgemeinen Krankenversicherung und zum Hausarztsystem vorgelegt, ohne dass nennenswerte Forschritte erzielt worden wären.

\subsection{Gesundheitsreform 1999}

Mit der Verabschiedung des Artikelgesetzes Nr. 4447 im August 1999 wurde eine umfassende Sozialreform in Gang gesetzt. ${ }^{43}$ Basierend auf den Vorgaben des 7. Fünfjahresplanes wurden für den Gesundheitsbereich die folgenden Eckpunkte vereinbart:

- Systemwechsel zur Allgemeinen Krankenversicherung

- Trennung von Leistungsfinanzierung und -erbringung

- Privatisierung der Krankenhäuser

- Einführung des Hausarztmodells

Angesichts der desolaten Finanzlage des Staates und der Sozialversicherungsträger wurden etliche Leistungen gestrichen, beispielsweise im zahnmedizinischen Bereich. So konnten die Gesundheitsausgaben zeitweise reduziert werden, indem Patienten mit höheren Zuzahlungen bei Arzneimitteln und Krankenhausaufenthalten belastet wurden. Schliesslich wurde mit der Absicht die starren Versorgungsstrukturen im türkischen Gesundheitswesen zu überwinden, die gegenseitige Öffnung der Versorgungsbereiche weiter forciert. Von gesundheitspolitischer Bedeutung war das Gesetz Nr. 4447 auch insofern, als Arbeitslose erstmals gegen Krankenheit abgesichert wuden. Insgesamt blieb die Gesundheitsreform 1999 aber bei zahlreichen Einzelmaßnahmen mit reinem Kostendämpfungscharakter.

\subsection{Bewertung}

„Die neunziger Jahren waren das Krisenjahrzehnt des türkischen Sozialsystems. " ${ }^{44}$ Nachdem in den neunziger Jahren mehrere Anläufe zur Gesundheitsreform gescheitert waren, kam mit der Gesundheitsreform 1999 erstmals wieder Bewegung ins Spiel. Mit ihr wurden die Bemühungen um Kostendämpfung erstmals in größerem Umfang in die Tat umgesetzt: Nämlich Leistungskürzungen bei gleichzeitiger Erhöhung der Zuzahlungen. Dies allein war bereits revolutionär und führte in der Folgezeit zu einem tiefgreifenden Umdenkprozess. Die EU-Beitrittsperspektive wirkte als Reformkatalysator. Das wurde besonders deutlich als der Europäische Rat im Dezember 1999 der Türkei offiziell den Status eines Beitrittskandidaten verlieh. Ankara ist seitdem bemüht, durch entsprechende Reformen die türkischen Verhältnisse an den eu- 
ropäischen Standard anzupassen. Trotz ihres Anspruchs, eine grundlegende Strukturreform einzuleiten, hatte die Reform in erster Linie Kostendämpfungscharakter. Sie bekämpfte nur die Symptome und ließ die Ursachen der Probleme, die hauptsächlich struktureller Art sind, weitgehend unberührt. Zudem wurde der Reformprozess von der schweren Finanzkrise überschattet, die das Land im Februar 2001 heimsuchte und die allein in den ersten Monaten weit über eine Million Arbeitsplätze forderte. Der Staatsbankrott konnte nur durch die Einschaltung des IWF in letzter Minute abgewandt werden. ${ }^{45}$ Gegen Auflagen wurde ein Kredit in Höhe von 16 Milliarden US-Dollar bereitgestellt. Es folgten zahlreiche Strukturreformen in der türkischen Wirtschaft: Alle mit dem Ziel, die Liberalisierung auch in bislang weitgehend verschonte Bereiche wie z.B. Telekommunikation, Energie, Landwirtschaft und letztlich auch in die Sozialsysteme hineinzutragen. Nahezu jede türkische Regierung, die in den neunziger Jahren an die Macht kam, betonte die dringende Notwendigkeit nach strukturellen Reformen im Gesundheitswesen. Bisher, so scheint es, ist nur die islamisch-konservative AKP im Begriff, dem gerecht zu werden.

\section{Gesundheitspolitischer Kurswechsel unter der AKP-Regierung}

Bei den vorgezogenen Parlamentswahlen vom 03.11.2002 ging die AKP unter Recep Tayyip Erdogan als Sieger hervor. Bereits ein Jahr später präsentierte sie ihr Gesundheitsreformprogramm unter dem bezeichnenden Titel Programm für einen Richtungswechsel im Gesundheitswesen (»Saḡlıkta dönüsüm Programis»), das mit finanzieller Unterstützung der Weltbank ab 01.01.2007 umgesetzt werden soll. Inhaltlich unterscheiden sich die Reformziele der AKP im Gesundheitswesen kaum von der seiner Vorgänger: Übergang zur Allgemeinen Krankenversicherung, Einführung des Hausarztmodells und insgesamt mehr Wettbewerb im System bilden die zentralen Themen. Neu sind die Rahmenbedingungen und der absolute Wille der AKP nach struktureller Veränderung. Seit November 2004 läuft im Primärbereich das Pilotprojekt zur Einführung des Hausarztmodells als Vorbereitung für die landesweite Ablösung des Gesundheitsposten-Modells durch den niedergelassenen Hausarzt in freier Praxis.

\subsection{Die Auflösung des Versorgungsmonopols der SSK}

Kennzeichnend für das türkische Gesundheitswesen ist seine duale Struktur. Die Master-Plan-Studie zum Gesundheitssektor offenbarte erstmals die Erkenntnis, dass die Hauptprobleme im türkischen Gesundheitswesen daraus erwachsen, dass sich zwei ökonomische Systeme mit gänzlich unterschiedlicher Logik überschneiden. Sie entspricht weder ökonomischer Sachlogik noch ist sie human. Sie ist vielmehr das Resultat historischer Entwicklung und der wirtschaftlichen Interessen einzelner Gruppen, allen voran der Beamten- und Arbeitnehmerschaft. ${ }^{46}$ Die AKP-Regierung hat in ihrem 8. Absichtsbrief an den
IWF für den Gesundheitsbereich den Systemwechsel zu einem umfassenden Beitragsmodell erklärt und in diesem Zusammenhang den Rückzug der SSK aus der medizinischen Leistungserbringung angekündigt. ${ }^{47}$ Mit der Übernahme der SSK-Versorgungseinrichtungen durch das Gesundheitsministerium am 19.02.2005 wurde der duale Charakter des türkischen Gesundheitswesens offiziell beendet.

\section{2 „Staatlich organisierte Entstaatlichung" als Ziel}

Bisweilen dominiert der türkische Staat die Gesundheitsversorgung. Er besitzt den Großteil der Gesundheitsinfrastruktur des Landes, deren Planung, Steuerung und Überwachung zentral durch das Gesundheitsministerium in Ankara erfolgt. Konkret sind das knapp $60.000 \mathrm{Be}-$ schäftigte in rund 700 Krankenhäusern, 6.000 Gesundheitsposten, 12.000 Gesundheitshäusern und zahlreichen weiteren Einrichtungen. Die gesundheitliche Versorgung der türkischen Bevölkerung erfolgt größtenteils innerhalb des staatlichen Gesundheitsapparates. Die Übernahme der SSK-Versorgungseinrichtungen ist nicht vergleichbar mit der staatlichen Einflußnahme ab 1961. Sie dient vielmehr als Vorarbeit für eine großangelegte Dezentralisierungsstrategie im Gesundheitswesen, die auch als staatlich organisierte Entstaatlichung bezeichnet werden kann. Die Leistungserbringung wird künftig im Rahmen eines Vertragsmodells „Dritten“ überlassen, die die Dienstleistungen auf einem Markt für Gesundheitsleistungen anbieten. Damit verbunden ist die Schaffung neuer Koordinierungs- und Entscheidungsinstanzen auf regionaler Ebene, die flexibel und zeitnah auf veränderte Umweltbedingungen reagieren sollen. Das Gesundheitsministerium widmet sich in Zukunft verstärkt globalen Aufgaben, indem es die Grundsätze der nationalen Gesundheitspolitik entwickelt und die Standards der medizinischen Versorgung festlegt. Seine Zuständigkeit in der unmittelbaren medizinischen Versorgung wird sich auf rein präventive Maßnahmen beschränken (z.B. Impfungen, Aufklärung). Damit kehrt es teilweise zurück zu seinem ursprünglichen Aufgabensprektrum in der Anfangsphase der Republik.

\subsection{Bewertung}

Mit ihrer positiven Haltung zum EU-Beitritt der Türkei unterscheidet sich die AKP von allen früheren islamistischen Parteien des Landes. Und anders als die Vorgängerregierungen hat die AKP mit ihrer absoluten Mehrheit im Parlament das nötige Potential, um nach Jahrzehnten des Reformstillstandes verkrustete Strukturen aufzubrechen und damit den Weg frei zu machen für ein effizientes und bürgernahes Gesundheitssystem. Langfristig geht es darum, die Stellung des Gesundheitsministeriums als Monopolanbieter von Gesundheitsdienstleistungen zu beseitigen. Dazu ist sie in zweifacher Hinsicht gezwungen: Einmal durch die EU, die von Ankara die Anpassung seiner gesundheitlichen Bestimmungen an die Normen 
der Europäischen Union verlangt und mehr noch seitens des IWF, deren gegenwärtig größter Schuldner die Türkei ist. Die Wirtschaftspolitik der AKP steht unter dem Diktat des IWF, der als „Heilmittel“ zur Sanierung der türkischen Wirtschaft die Privatisierung der staatlichen Unternehmen und Monopole fordert. Einen kleinen, wenn auch wichtigen Teilerfolge hat die AKP bereits mit der Ausschaltung der SSK aus dem medizinischen Versorgungsgeschäft erzielt. Weitere Herausforderungen, insbesondere der Abschied vom Beamtenstatus im Personalbereich oder die Entlassung der staatlichen Krankenhäuser in ein wettbewerbliches Umfeld unter Eingliederung in die jeweiligen kommunalen Versorgungsstrukturen, stehen noch bevor.

\section{Zusammenfassung und Ausblick}

Die Türkei blickt auf eine über 80-jährige Geschichte in der Gesundheitspolitik zurück. Sie ist geprägt durch das allmähliche Eindringen des Staates in die Gesundheitsversorgung. Seit der Republikgründung 1923 hat sich die Bevölkerung des Landes nahezu verfünffacht, ohne dass jedoch die medizinische Versorgung seither hinreichend gelöst worden wäre. Während anfangs die Bekämpfung ansteckender Krankheiten im Mittelpunkt des staatlichen Interesses stand, rückte mit der zunehmenden wirtschaftlichen Entwicklung des Landes die Gesundheitsversorgung der breiten Bevölkerung ins staatliche Visier. Aus der Überzeugung, dass nur der Staat das gesundheitspolitische Ziel „Gesundheit für alle“ gewährleisten könne, wurde nach 1961 im Zuge der Sozialisierung ein umfassendes Netz an ambulanten und stationären Versorgungseinrichtungen errichtet. Seit Mitte der achtziger Jahre wird über eine grundlegende Strukturreform des türkischen Gesundheitswesens nachgedacht. Der staatliche Versorgungsapparat gerät seither immer stärker wegen „angeblich“ mangelnder Leistungsfähigkeit ins Visier neoliberaler Privatisierungsbefürworter, ohne dass der Blick hinreichend auf die systematische Unterfinanzierung des Systems seitens der Politik als Ursache gerichtet wird. Das Budget des Gesundheitsministeriums wurde insbesondere während der neunziger Jahre laufend gekürzt. Lag sein Anteil 1993 noch bei 4,6\% des gesamten Staatshaushaltes, so schrumpfte er bis 2003 auf 2,3\%. Knapp 90 \% des Budgets wird gegenwärtig zur Deckung der Personalkosten verwendet, womit praktisch kein nennenswerter finanzieller Spielraum für Investitionen in die Gesundheitsinfrastruktur übrig bleibt (Investitionsstau). Der medizinische Fortschritt, die steigende Lebenserwartung sowie nicht zuletzt der Zwang zur Haushaltskonsolidierung machen eine Reform des Gesundheitswesen unumgänglich.

Angesichts leerer Kassen greift der türkische Staat heute - anders als vor über 50 Jahren - auf eine neue Zauberformel zurück, um das Ziel „Gesundheit für alle“ zu verwirklichen: Öffentlich-private Partnerschaften (engl. Private Public Partnerships - PPP). Anders als bei einer vollständigen Privatisierung bleibt die öffentliche Hand bei dieser Strategie weiter verantwortlich für die Inhalte der Da- seinsvorsorge, während die Erbringung für die Bürger in Kooperation mit einem privaten Partner erfolgt. ${ }^{48}$ Mit der aktuellen Gesundheitsreform sind die Beteiligten im Gesundheitswesen gezwungen, sich von Verwaltungsinstanzen zu Dienstleistern zu wandeln, Macht abzugeben und fähig zu werden, sich in einer dynamischen Umwelt stets immer wieder neu zu entwerfen. Damit wird ein vielfältiges und weitreichendes Repertoire an Techniken und modernen Steuerungsinstrumenten zum Einsatz gelangen, angefangen von den Diagnosis Related Groups ${ }^{49}$ (DRG) über Disease Management Programme (DMP) bis hin zu Bonusprogrammen für gesundheitsbewusstes Verhalten. Der EU-Beitrittskandidat Türkei gilt zudem als ein wichtiger (strategischer) Aspirant auf dem künftigen europäischen Gesundheitsmarkt. Insbesondere aus deutscher Sicht dürfte dies mit Blick auf die rund zweieinhalb Millionen türkischstämmigen Migranten im Land enorme Chancen im Bereich der grenzüberschreitenden Gesundheitsversorgung eröffnen.

Die Entwicklung des türkischen Gesundheitswesens entlang berufsgruppenbezogener Sicherungssysteme in Verbindung mit einem finanziell unterversorgten staatlichen Gesundheitsdienst versperrte für Millionen von Menschen im Land systematisch den Zugang zur medizinischen Versorgung. Ob das neue Gesundheitssystem der Türkei mit der Kombination aus beitragsfinanzierter Krankenversicherung und der Einbeziehung des privaten Sektors in die Leistungserbringung im Rahmen des Privat Public Partnership dabei - angesichts der extrem ungleichen Einkommensverteilung, Arbeitslosigkeit sowie Schwarzarbeit - der richtige Weg sein wird, den Zugang zu essenzieller Gesundheitsversorgung sicherzustellen bleibt abzuwarten. Eines ist jedoch sicher, nämlich, dass die Türkei gegenwärtig seine bislang weitreichendste Gesundheitsreform nach 1961 erlebt.

\section{Literatur}

Aşkın, Basri (2002): Von der Selbstkostendeckung zu den „Diagnosis Related Groups“ (DRGs) - Hintergründe, Grundlagen und Auswirkungen der DRG-Einführung in Deutschland ab 2003, Marburg 2002.

Așkın Basri (2004): das Sozialversicherungssystem der Türkei. Rechtliche Grundlagen, Strukturen, Reformperspektiven, Marburg 2004.

Așkın, Basri (2004a): Typen sozialer Sicherung in der EU - Eine Positionsbestimmung am Beispiel der Türkei, in: Zeitschrift für Türkeistudien (ZfTS), 17. Jhrg., Heft 1+2, S. 245 - 254, Essen 2004.

Așkın, Basri (2004b): Die Grundzüge des türkischen Sozialversicherungssystems, in: Die Betriebskrankenkasse (Die BKK), 92. Jhrg., Heft 11, S. 510 - 513, Essen 2004.

Aşkın, Basri (2005): Das türkische Gesundheitswesen im Umbruch. Die Übernahme der SSK- Krankenhäuser durch das türkische Gesundheitsministerium, in: Zeitschrift für internationales und ausländisches Arbeits- und Sozialrecht (ZIAS), 19. Jhrg., Heft 2, S. 204-209, Heidelberg 2005.

Asskın, Basri (2006): Alterssicherung in der Türkei: Grundlagen, Strukturen, Reformperspektiven - Beitrag anlässlich der Arbeitstagung der Deutschen Rentenversicherung Bund zur „Alterssicherung im internationalen Vergleich" vom 19.10.2005 bis 21.10.2005 in Erkner - in: Deutsche Rentenversicherung, Heft 1, 61. Jhrg., Berlin 2006.

Elger, Ralf / Stolleis, Friederike (2001): Kleines Islam-Lexikon. Geschichte - Alltag - Kultur, München 2001.

Ferner, Manfred (2004): KulturSchock Türkei, Bielefeld 2004. 
Schirrmacher, Gerd (1983): Sozialwesen und Sozialarbeit in der Türkei: Bedingungen, Konzepte, Organisationsformen und Praxis in ausgewählten Praxisfeldern, Köln 1983.

Stolpe, Manfred (2006): Investitionen mobilisieren, staatliches Handeln modernisieren, in: Pauly, Lothar (Hrsg.): Das neue Miteinander. Public Private Partnership für Deutschland, Hamburg 2006, S. 65-71.

\section{Fußnoten}

1 Das Aufgabenspektrum der Stiftungen umfasste z.B. im religiösen Bereich etwa den Bau und die Erhaltung von Moscheen, im kulturellen Bereich die Unterhaltung von Schulen, Medresen und Bibliotheken, im mildtätigen Bereich die Hilfen für Bedürftige sowie den Bau sozialer Einrichtungen (Krankenhäuser, Sanitätsstationen etc.). Vgl. Elger / Stolleis (2001), S. 78. Für einen ausführlichen Einblick in das islamische Sozialwesen siehe insbesondere Schirrmacher (1983).

2 Bis auf zwei kleinere Ausnahmen wurde die Türkei bis 1945 durch die von Atatürk gegründete Republikanische Volkspartei (CHP) regiert, die primär aus der militärischen Führung des Befreiungskrieges hervorgegangen war (Ein-Parteien-Regierung).

3 Als Balkan-Türken werden jene Türken bezeichnet, die sich im Zuge der Expansion des Osmanischen Reiches seit dem 14. Jahrhundert im europäischen Teil des osmanischen Herrschaftsgebietes angesiedelt haben.

4 Die Verabschiedung des Allgemeinen Gesundheits- und Hygienegesetzes mit der Nummer 1593 v. 24.04.1930 wurde von der Entdeckung der Bakterien als Krankheitsverursacher und vom medizinischen Fortschritt zu Beginn des 20. Jahrhunderts nachhaltig beinflusst.

5 Auf der Grundlage des Allgemeinen Gesundheits- und Hygienegesetzes wurde auch der „Oberste Gesundheitsrat“ der Türkei (»yüksek saḡlık şurası«) gegründet. Als fachliches Beratungsgremium äußert er seine Meinung zu medizinischen Themen und unterbreitet entsprechende Vorschläge.

6 Auf Bezirksebene waren die jeweiligen Landräte (»Kaymakam«) und auf Provinzebene die Provinzgouverneure $(»$ Vali«) verantwortlich für die Gesundheitsversorgung. Sie leiteten zugleich die Kommissionen.

7 Eine Ausnahme bildeten die sog. kurativen Behandlungshäuser (»Muayene ve Tedavi Evleri«), die auf Beschluss der Regierung im Jahre 1924 in einigen Städten errichtet worden sind.

8 Vgl. Schirrmacher (1983), S. 137.

9 Grundlage bildete das Gesetz Nr. 3017 v. 09.06.1936 mit dem alle (primär präventiven) Versorgungseinrichtungen, sowie einschliesslich der medizinisch-pflegerischen Ausbildungseinrichtungen, dem Ministerium für Gesundheit und soziale Hilfe unterstellt wurden. Zudem erhielt das Ministerium neue Organisationseinheiten wie z.B. in den Bereichen Gesundheitserziehung und medizinische Statistik.

10 Regierungsärzte gab es bereits vor 1923. Sie sind ein wichtiger Beleg für die beginnende Entwicklung der staatlich organisierten Gesundheitsdienste im Osmanischen Reich gegen Ende des 19. Jahrhunderts. Refik Saydam übernahm das Konstrukt der Regierungsärzte und setzte sie beim Aufbau der medizinischen Versorgung des Landes ein.

11 Die rechtliche Grundlage zum Bau der Schule für öffentliche Gesundheit geht zurück auf das bereits erwähnte Gesetz Nr. 3017 aus dem Jahre 1936

12 Die türkische Ärzteschaft musste sich zu dieser Zeit weder gegen staatliche Interessen noch gegen etwaige Krankenkassen behaupten. Dieser Umstand erklärt auch, weshalb der Anstoß für die Organisierung der ärztlichen Interessen im Land gerade von einem nationalen Gesetz ausging.

13 Vgl. Schirrmacher (1983), S. 654.

14 Daran änderte auch die Tatsache nichts, dass das Türkische Bürgerliche Gesetzbuch von 1926 innerhalb der Familie gleiche Rechte für Mann und Frau vorsah. Bildung und Aufklärung des Volkes waren deshalb für Atatürk die Kernpunkte der Mobilisierung und Modernisierung der Türken.

15 Die Arbeitsunfallkrankenhäuser bildeten die ersten Gesundheitseinrichtungen der Arbeiterversicherungsanstalt.

16 Während des gesamten Zweiten Weltkrieges hat die Türkei unter Ismet Inönü (1884-1973), dem Nachfolger Atatürks, eine Politik der Neutralität verfolgt und erst gegen Kriegsende Deutschland den Krieg erklärt.

171952 trat die Türkei dem Nordatlantischen Verteidigungspakt (NATO) bei.

18 Als Haupthindernis, die eine Umsetzung letztlich verhinderte, wurden insbesondere die enormen Kosten im Falle einer landesweiten flächendeckenden Einführung der Krankenversicherung angeführt.
19 Diese Problematik ist heute noch nach wie vor aktuell und betrifft die gegenwärtig geplante Einführung der Allgemeinen Krankenversicherung in der Türkei analog.

20 Für nähere Einzelheiten zu diesem staatlichen Versicherungsträger sowie allgemein zum türkischen Sozialversicherungssystem siehe Asskın (2004).

21 Die Städtischen Krankenhäuser waren ursprünglich mit dem Ziel gegründet worden, die medizinische Versorgung der in den Kreisverwaltungen tätigen Beamten und ihrer Angehörigen sicherzustellen. Nach 1970 wurde der Personenkreis sukzessiv erweitert auf Bedürftige, Selbstständige und Selbstzahler. Die Finanzierung dieser Städtischen Einrichtungen erfolgt unmittelbar aus der Stadtkasse.

22 Die bereits früher im Gesetz Nr. 4772 enthaltenen Vorschriften zur Mutterschaftsversicherung wurden dabei teilweise modifiziert und in das Gesetz Nr. 5502 integriert.

23 Die türkische Ärzteschaft verfolgte mit diesem Schritt nicht zuletzt das Ziel sich vom direkten Staatszugriff zu emanzipieren. Mit der Verstaatlichung der Krankenhäuser wurde der ärztliche Handlungsspielraum freilich erheblich eingeschränkt.

24 Durch die konsequente Arbeit der Tuberkulosebekämpfungsvereine (»Verem Savas Dernekleri«), die der Staat 1949 in mehreren Städten errichtet hatte, konnte ein deutlicher Rückgang der gemeldeten Seuchenerkrankungen verzeichnet werden.

25 Vgl. Ferner (2004), S. 37.

26 Als Beispiel garantierte die Verfassung von 1961 erstmals das Streikund Demonstrationsrecht und ließ die Presse- und Meinungsfreiheit $\mathrm{zu}$.

27 Die Verfassung von 1961 sah die Verwirklichung von Fortschritt und Wachstum durch konsequente Einhaltung der Fünfjahrespläne vor (mixed economy). Zu diesem Zweck wurde im Jahre 1960 die staatliche Planungsbehörde (DPT) errichtet. 1963 trat der erste Fünfjahresplan in Kraft. Die Pläne haben seither für den staatlichen Sektor verbindlichen, für den privaten Sektor dagegen lediglich empfehlenden Charakter.

28 Zum öffentlichen (staatlichen) Gesundheitswesen wird im vorliegenden Beitrag nur das Versorgungssystem des Gesundheitsministeriums subsummiert, weil nur auf diesen das Kriterium des „öffentlich zugänglichen“ zutrifft. Im Fall des arbeitnehmereigenen Versorgungssystems erfolgt die Einbindung nicht in der Rolle des Staatsbürgers, sondern rein über das Arbeitsverhältnis.

29 Die Gesundheitsdienste umfassen dem Gesetz Nr. 224 zufolge sämtliche Maßnahmen, die der Förderung, dem Erhalt und der Wiederherstellung der physischen und psychischen Gesundheit dienen $(\$ 2 b)$.

30 Ein derart umfassendes Verständnis von Gesundheit war in der Türkei bis dahin unbekannt. Lange vor der Proklamation des Primary Health Care-Konzeptes in Alma Ata im Jahre 1978 wurde zudem anerkannt, dass eine Verbesserung der Gesundheit nicht rein durch kurative Maßnahmen erreichbar ist, sondern dass Gesundheit vielmehr nur durch einen intersektoralen, primär präventiven, an den Grundbedürfnissen und der Armutsbekämpfung orientierten Ansatz verbessert werden könne.

31 Die Investitions- und Personalkosten werden nahezu überwiegend durch die öffentliche Hand getragen. Der überwiegende Teil der Betriebskosten hingegen erfolgt zu fast gleichen Anteilen aus Steuern und Beiträgen und zu einem geringeren Teil aus Eigenbeteiligungen. „Fremdentgelte“ in Form von Beiträgen und Eigenbeteiligungen stellen eine wichtige Finanzierungsquelle für die Aufrechterhaltung des staatlichen Versorgungssystems dar.

32 Durch die Einschreibung in einem Gesundheitsposten sollten die Einwohner im Einzugsgebiet der jeweilgen Gesundheitsposten ursprünglich kostenlos medizinisch versorgt werden.

33 Die vertikal strukturierten Seuchenbekämpfungsdienste aus den Anfängen der Republik wurden zum Teil als Dispensaire in das Gesundheitsposten-Modell integriert.

34 Als Querschnittsaufgabe wird Familienpolitik in der Türkei heute überwiegend durch das Gesundheitsministerium wahrgenommen.

35 Gezeichnet durch die hohen Bevölkerungsverluste (sowohl während des Ersten Weltkrieges, als auch durch den nationalen Befreiungskrieg) lag bis in die sechziger Jahre hinein das Ziel der türkischen Bevölkerungspolitik in der Forcierung eines raschen Bevölkerungswachstums

36 Dahinter stand auch weniger die Absicht die Frauen zu stärken, sondern die Familie als Einheit.

37 Vgl. auch Asskin (2004a), S. $250 f$.

38 Vgl. Așkın (2004), S. $16 f$.

39 In der Praxis spielen Gesundheitsförderung und Prävention allerdings kaum eine Rolle. Anders als im Gesetz Nr. 224 beschränkte sich der 


\section{THEMA}

Wirkungskreis des Gesetzes Nr. 506 auf die einzelnen Betriebe. Die SSK schenkt den Lebensumständen der Arbeitnehmer ausserhalb des Betriebs traditionell wenig Aufmerksamkeit.

40 Trotz gegenseitiger Abschottungsversuche gab es immer wieder gemeinsame Versorgungsverträge. So z.B., wenn es um die medizinische Versorgung von SSK-Versicherten in Regionen ging, wo die SSK über keine eigene Versorgungsinfrastruktur verfügte.

41 In Aşkın (2004a) erfolgt der Versuch das türkische Gesundheitswesen in eine der vier Typen der sozialen Sicherung in Europa einzuordnen, namentlich in die Typen des kontinentaleuropäischen, angelsächsischen, skandinavischen sowie südeuropäischen Sicherungsmodells.

42 Die Förderung und Ausweitung des privaten Gesundheitssektors, sowie der Übergang zu einem umfassend beitragsfinanzierten Krankenversicherungssystem waren bereits im, den Zeitraum von 1985 bis 1989 umfassenden, 5. Fünfjahresplan, als Ziele explizit formuliert.
43 Die Einführung der Arbeitslosenversicherung in der Türkei erfolgte ebenfalls durch dieses Gesetz. Für nähere Einzelheiten siehe in A_kın (2004) sowie auch in A_kin (2004b).

44 A_kin (2006), S. 63.

45 Vgl. auch Asskin (2006), S. 68.

46 Das im Zuge der Einführung der Arbeiterkrankenversicherung sukzessiv ausgebaute medizinische Versorgungsnetz der SSK entwickelte sich (entgegen dem staatl ichen Gesundheitsdienst) völlig unabhängig von internationalen Einflüssen.

47 Eine ausführlichere Beschreibung der Hintergründe und Ziele dieser Übernahmestrategie ist in A_kın (2005) zu finden.

48 Vgl. Stolpe (2006), S. 67.

$49 \mathrm{Zu}$ den Diagnosis Related Groups (DRGs) siehe ausführlich in Așkın (2002).

\section{"Standardausstattung"}

Prof. Dr. Tobias Fröschle, www.socialnet.de

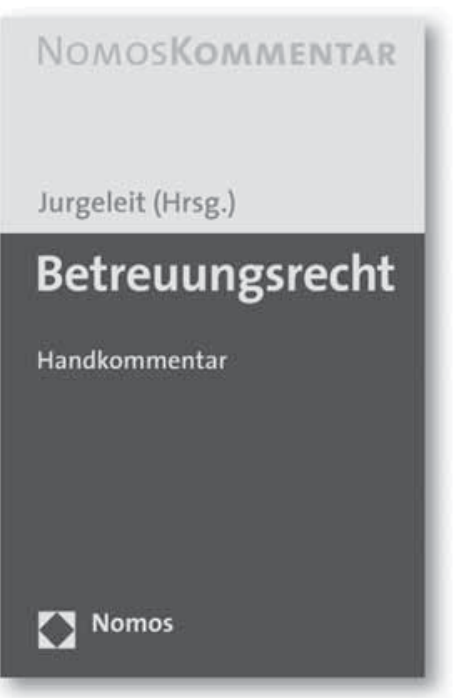

\begin{abstract}
Betreuungsrecht
Handkommentar

Herausgegeben von RiOLG Dr. Andreas Jurgeleit, Hamm

2006, 704 S., geb., 69,- $€$, ISBN 978-3-8329-1367-0
\end{abstract}

"Überzeugend ist bei diesem Kommentar die Praxisnähe, weshalb er sowohl für ehrenamtliche Betreuer als auch für Berufsbetreuer, Richter, Verfahrenspfleger und Betreuungsbehörden bestens geeignet ist." RA Markus Band, FGPrax 6/06

"Handelt es sich um eine fundierte Aufbereitung der grundlegenden Vorschriften des Betreuungsrechtes, die auch für die örtlichen Betreuungsbehörden von großem Nutzen sein kann. Es ist dem Kommentar zu wünschen, dass er sich zu einer verlässlichen Hilfe für die örtliche Praxis entwickelt."

Dr. Irene Vorholz, Berlin, Der Landkreis 10/06 